\title{
Fifth day fits: an acute zinc deficiency syndrome?
}

\author{
H JAMES GOLDBERG AND ELIZABETH M SHEEHY
}

Mercy Maternity Hospital, Melbourne, Australia

\begin{abstract}
SUMMARY A 3-year prospective study to elucidate the cause of fifth day fits was carried out. A highly significant relationship with low cerebrospinal fluid zinc levels was found. Theoretical and practical considerations support the contention that fifth day fits are caused by an acute deficiency of zinc.
\end{abstract}

Convulsions occurring around the fifth day of life in otherwise normal babies have aroused considerable interest ${ }^{1}$ because of an apparent rise in incidence and also because the aetiology is unknown. Our hospital excerience ${ }^{2}$ during the periods 1971-74 (12 904 live births) and 1975-77 (13 304 live births) showed a rise in the overall incidence of neonatal convulsions from 2 to 6 per 1000 live births while the incidence of fifth day fits rose from 0.6 to $2 \cdot 2$ per 1000 live births. A prospective study was started in July 1978 to define the syndrome and identify possible causes.

\section{Method}

The fifth day fitters (group 1) were defined as being normal term babies of appropriate size for gestational age who developed short-lived and otherwise unexplained convulsions towards the end of the first week of life. They were compared with a group of fitting babies (group 2) in whom a cause for convulsions had been provisionally identified or in whom there was some other health problem (such as being small for gestational age) even if not obviously related to the convulsions. For further comparison a group of babies (group 3) was incorporated into the study in whom there had been no convulsions although lumbar punctures had been performed for other indications-for example suspicion of sepsis. From the start of the study all specimens of cerebrospinal fluid (CSF) from groups 1,2 , and 3 were frozen and stored until required for screening programmes.

Most $(87 \%)$ of the fifth day fitters were breast fed. This accurately reflects the pattern in our hospital where about $90 \%$ of term babies are wholly or primarily breast fed on discharge at day 7 .

All babies who convulsed had the following studies carried out: full blood examination, blood sugar, serum sodium, calcium, phosphate, magnesium, acid-base estimations, serum proteins, serum IgM, metabolic screen of urine and blood, CSF microscopical examination and bacterial culture, skull $x$-ray film. A full antenatal and perinatal drug history was recorded together with a postnatal drug and feeding history of both mother and baby. Initially all babies had viral cultures of CSF, blood, urine, and faeces, and either a computerised tomography scan or ultrasound examination of the brain. Because few results were significant these tests were later reserved for babies with specific clinical indication of viral infection or a structural brain abnormality. If clinically indicated further elucidation of metabolic abnormalities was done. Several babies had a trial of pyridoxine without success.

The time of onset of the fits suggested an environmental influence; furthermore, it was noted that while all the cases of fifth day fits came from the standard postnatal nurseries there appeared to be no such unexplained convulsions among babies in the special care nurseries. Comparison of nursing management between the two areas showed that those babies in the standard postnatal nurseries received smaller volumes of feeds, more exposure to Phisohex ( $3 \%$ hexachlorophane), and less exposure to zinc ointme.t than those in the special care nurseries. The last observation prompted us to study the levels of zinc in the CSF. Estimations of CSF zinc levels were carried out by an independent laboratory and were not identified to any group. Analysis was by flame-aspiration atomic absorption spectroscopy.

Investigation of the air-conditioning system throughout the hospital showed no source of infection or toxins. Testing for hexachlorophane showed no detectable amounts in the CSF specimens of any in the three groups. Attempts at screening specimens of CSF for possible toxins using liquid gas chromatography were unsatisfactory because of difficulties in developing standard curves for all the potential toxins.

\section{Results}

CSF zinc levels were examined (Figure) and a nonparametric comparison made between group 1 (fifth day fitters) and groups 2 and 3 (controls) based on the number of CSF zinc levels above or below $1 \mu \mathrm{mol} / \mathrm{l}$. (With the skew distribution for CSF zinc levels it is not appropriate to use a $t$ test or analysis 


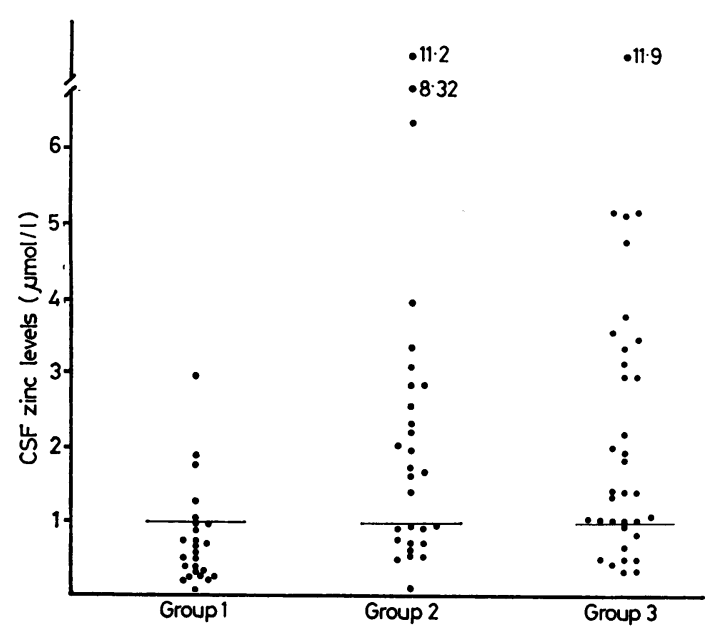

Figure Cerebrospinal fluid zinc levels. Group 1 fifth day fitters; group 2 convulsions due to some other cause; group 3 CSF samples from babies who did not fit but who had other health problems.

of variance to compare group means.) Thus only $5(20 \%)$ of 25 bab:es in group 1 had CSF zinc levels above $1 \mu \mathrm{mol} / \mathrm{l}$ compared with $41(64 \%)$ of 64 babies in groups 2 and 3. Analysis of this $2 \times 2$ table showed a highly significant tendency $\left(\chi^{2},>14 \cdot 0\right.$, $\mathbf{P}<0.0001$ ) for CSF zinc levels to be lower than $1 \mu \mathrm{mol} / 1$ more often in group 1 than in groups 2 and 3 (relative risk $=8 \cdot 2$ ). A normal mean CSF zinc level has been given as $3.7 \mu \mathrm{mol} / 1 .^{3}$

\section{Discussion}

These data suggest that unexplained fits occurring around the fifth day may be caused by transient zinc deficiency. Although zinc deficiency has not hitherto been recognised as a cause for convulsions, certain neurological abnormalities ${ }^{4}$ have been ascribed to zinc deficiency, most of them of a chronic nature. These include loss of taste and smell, depression, irritability, behavioural abnormalities, nystagmus, and optic neuritis. Perhaps an acute deficiency of zinc would have more striking manifestations.

Absorption of zinc in the newborn is believed to be facilitated by zinc-binding ligands in the breast milk which diminish after a few days and zincbinding ligands in the gut mucosa which take some days to be fully developed. ${ }^{5}$ Under these circumstances zinc absorption is likely to be lowest towards the end of the first week of life. Zinc is better absorbed from breast milk than from artificial feeds.
However, variations in the quality and quantity of the milk ingested are far greater in breast-fed than artificially-fed infants and may account for further variation among babies. ${ }^{6}$ The level of calcium in the feeds also affects the absorption of zinc, with high concentrations of calcium having an inhibitory effect. It is not known whether greater volumes of feeds or external applications of zinc ointment give any protection against acute zinc deficiency. With further development $o$ mucosal zinc-binding ligands in fifth day fitters, improved absorption of zinc with spontaneous resolution of symptoms might be expected.

We did not attempt to reverse the syndrome by giving zinc; indeed it may be difficult to prove that zinc defisiency is a cause of fifth day fits, firstly because the convulsions are short-lived, and secondly because it is not known how long it takes for either orally or parenterally administered zinc to become biologically available in the central nervous system. As the fits respond readily to standard treatment the use of experimental measures was not justified. As plasma zinc levels vary with the serum albumin level and fall within hours of stress it was felt that CSF levels of zinc were a more accurate reflection of 'neurologically available' zinc than were plasma estimations. No other types of zinc estimations were carried out. Parameters such as white blood cell zinc are thought to be a more accurate reflection of total body zinc than plasma or CSF levels. However, their relevance in this context is unknown, particularly as the proportion of zinc in the various body compartments may change rapidly over the first weeks of life. ${ }^{7}$

Further studies are planned to correlate zinc levels in artificial feeds and human milk with the occurrence of fits, to investigate the relevance of concomitant calcium levels in the feeds, and to assess the affects of feeding schedules and of the external applications of zinc cream on the incidence of the syndrome.

We thank Dr L I Taft for laboratory assistance and generous financial support and Dr J D Mathews for the statistical analysis and review of the manuscript.

\section{References}

1 Pryor D S, Don N, Macourt D C. Fifth day fits: a syndrome of neonatal convulsions. Arch Dis Child 1981; 56: 753-8.

2 Goldberg H J. Convulsions. In: Beischer N, Abell D A, eds. Second clinical Report: Mercy Maternity Hospital, Melbourne. North Melbourne: Ramsay, Ware, Stockland, 1978: 157.

3 Lyengan G V, Kollmer W E, Bowen H J M, eds. 
Elemental composition of human tissues and body fluids. Weinheim: Verlag Chemi, 1978: 38.

4 Henkin R I, Patten B M, Re P K, Bronzer D A. A syndrome of acute zinc loss. Arch Neurol 1975; 32: 745-51.

5 Shaw J C L. Trace elements in the fetus and young infant. Am J Dis Child 1979; 133: 1260-8.

6 Picciano M F, Guthrie H A. Copper, iron, and zinc contents of mature human milk. Am J Clin Nutr 1976; 29: $242-54$.
7 Aggett P J, Harries J T. Current status of zinc in health and disease states. Arch Dis Child 1979; 54: 909-17.

Correspondence to Dr H J Goldberg, Mercy Maternity Hospital, Clarendon Street, East Melbourne, Victoria 3002, Australia.

Received 22 March 1982

\title{
Renal pseudotumours caused by xanthogranulomatous pyelonephritis
}

\author{
A R WATSON, H B MARSDEN, M LENDON, AND P H MORRIS JONES \\ Royal Manchester Children's Hospital, and Booth Hall Children's Hospital, Manchester
}

SUMMARY Five cases of xanthogranulomatous pyelonephritis are described. Wilms's tumour was suspected preoperatively in 2 of the cases and a renal tumour appeared to be confirmed at laparotomy in three. Patho ogical examination is diagnostic and the condition appears to be always unilateral. Nephrectomy is curative.

Xanthogranulomatous pyelonephritis (XPN) is a rare outcome of bacterial infection of the kidney, characterised by unilateral destruction of renal parenchyma and the accumulation of lipid-laden foamy macrophages either surrounding abscess cavities or as discrete yellow nodules. Most cases have been described in women, generally they have a non-functioning kidney, urolithiasis, and urinary infection especially with Proteus species. The similarity of XPN to renal cell carcinoma has often been noted and the condition may also mimic perinephric abscess, pyelonephritis with stones, renal carbuncle, and tuberculosis. ${ }^{1}$

Some 45 cases of XPN have been documented since the first description in $1963 .^{2-6}$ We describe 5 more cases in order to stress the importance of considering XPN in the differential diagnosis of renal tumours.

\section{Patients}

Case 1. A 2-year-old boy was noticed at routine examination to be anaemic with weight below the 3rd centile and height on the 10th centile. A leftsided abdominal mass was palpable and an intravenous urogram (IVU) showed patchy calcification in the lower portion of the left kidney with irregular distortion and enlargement of the upper calyces. His haemoglobin concentration was 6.8 $\mathrm{g} / \mathrm{dl}$ with a white cell count of $13.8 \times 10^{9} / 1,72 \%$ neutrophils. Urine analysis showed pyuria but no growth on culture.

After blood transfusion laparotomy was performed for a suspected Wilms's tumour. This appeared to be confirmed at the time of operation with enlarged glands in the para-aortic and inferior mesenteric areas. Left nephrectomy and extensive lymph node dissection was performed. Vincristine $0.7 \mathrm{mg}$ was given intravenously at the end of the operation.

Histological examination of the nephrectomy specimen showed changes typical of XPN with abscesses, lipid-filled histiocytes, and chronic inflammatory cells. Proteus sp. were obtained on culture of the pus. The lymph nodes showed reactive hyperplasia. He remains well and free of urinary infection 4 years later with a weight on the 25th centile and height on the 10th centile.

Case 2. A $3 \frac{1}{2}$-year-old boy was admitted for investigation of small stature, anaemia, and poor appetite. His weight was below the 3rd centile and his height between the 3rd and 10th centiles. Abdominal examination showed a palpable right kidney. His haemoglobin concentration was 8.5 $\mathrm{g} / \mathrm{dl}$ with a white cell count of $19.8 \times 10^{9} / 1$. Urine analysis showed pyuria with no growth on culture. Calcification was noted in the right renal area on plain abdominal $x$-ray film and an IVU showed deformity of the right calyceal system in a kidney which was enlarged. Cystoscopy and cystogram showed no ureteric reflux. Operation for suspected pyelonephritis with stones was carried out. A large tumour mass was found which was adherent to the colon, duodenum, liver, and diaphragm with enlarged adjacent lymph nodes. Nephrectomy was performed and he was given vincristine $0.75 \mathrm{mg}$ intravenously in theatre. Pathological examination 